\title{
Photo-imprint super-resolution photoacoustic microscopy
}

Junjie Yao, Lidai Wang, Chiye Li, Chi Zhang, Lihong V. Wang

Junjie Yao, Lidai Wang, Chiye Li, Chi Zhang, Lihong V. Wang, "Photoimprint super-resolution photoacoustic microscopy," Proc. SPIE 9323, Photons Plus Ultrasound: Imaging and Sensing 2015, 932327 (11 March 2015); doi: $10.1117 / 12.2077350$

SPIE. Event: SPIE BiOS, 2015, San Francisco, California, United States 


\title{
Photo-imprint Super-resolution Photoacoustic Microscopy
}

\author{
Junjie Yao, Lidai Wang, Chiye Li, Chi Zhang, Lihong V. Wang* \\ Optical Imaging Laboratory, Department of Biomedical Engineering, \\ Washington University in St. Louis, St. Louis, MO 63130, USA \\ *Correspondence to: $\underline{\text { lhwang@wustl.edu }}$
}

\begin{abstract}
Combining the absorption-based photoacoustic effect and intensity-dependent photobleaching effect, we demonstrate a simple method for super-resolution photoacoustic imaging of both fluorescent and non-fluorescent samples. Our method is based on a double-excitation process, where the first excitation pulse partially and inhomogeneously bleaches the molecules in the diffraction-limited excitation volume, thus biasing the signal contributions from a second excitation pulse striking the same region. By scanning the excitation beam, we performed three-dimensional sub-diffraction imaging of varied fluorescent and non-fluorescent species. A lateral resolution of $80 \mathrm{~nm}$ and an axial resolution of 370 $\mathrm{nm}$ have been demonstrated. This technique has the potential to enable label-free super-resolution imaging, and can be transferred to other optical imaging modalities or combined with other super-resolution methods.
\end{abstract}

Keywords: super-resolution imaging, sub-diffraction imaging, photoacoustic microscopy, label-free, non-fluorescent imaging

\section{INTRODUCTION}

Diffraction has long limited optical microscopy to a lateral resolution of $\sim 200 \mathrm{~nm}$, leaving the observation of ultrastructural cellular features to electron microscopy. In recent years, super-resolution optical microcopy has broken the diffraction limit, enabling numerous observations of previously unresolvable cellular structures and processes at the level of tens of nanometers [1-4]. However, relying on the emission nonlinearity of fluorophores, most of the existing superresolution techniques perform only fluorescence imaging [5-8].

In the method we describe here, the resolution enhancement is based on the excitation nonlinearity of the photobleaching effect, a common phenomenon in optical imaging which is otherwise regarded as harmful $[9,10]$. The photobleaching effect depends strongly on the excitation intensity, which enables super-resolution imaging by spatially trimming the excitation volume to a sub-diffraction size [11-13]. Photoacoustic (PA) imaging, which acoustically probes optical absorption contrast in biological tissue, can potentially image all molecules [14]. The combination of the photobleaching effect and photoacoustic imaging can potentially achieve super-resolution imaging over a wide-range of species.

\section{METHODS}

The principle of our photo-bleaching-based photo-imprint photoacoustic microscopy (PI-PAM) is illustrated in Fig. 1 [15]. When a Gaussian-shape diffraction-limited excitation laser spot strikes on densely distributed absorbers, the absorbers are inhomogeneously bleached, depending on the local excitation intensity (Fig. 1a). The absorption reduction in the center of the excitation spot is greater than that in the periphery (Fig. 1b). As a result, when the second pulse excites the same region, the center portion contributes less to the second PA signal than the periphery. The difference between the two PA signals incorporates the absorption reduction distribution (Fig. 1c), sharpening the center of the focus.
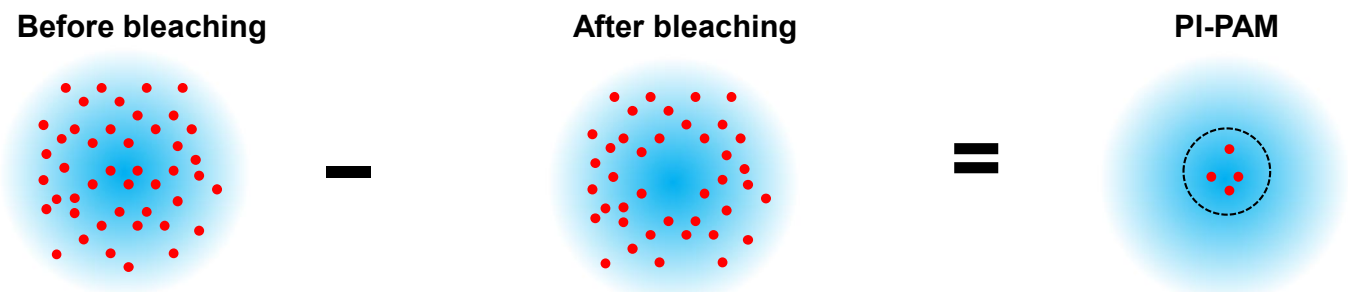

Figure 1. Principle of PI-PAM. Within the optical focal spot, the laser intensity has a Gaussian distribution with its FWHM of $\sim 200$ $\mathrm{nm}$. Since the photobleaching rate depends on the local excitation intensity, the first excitation bleaches the center part of the 
illuminated region more than the periphery, leaving an imprint in the sample. The differential signal between before- (left panel) and after-bleaching (middle panel) images results in a smaller effective excitation size and thus a resolution enhancement, as shown by the dashed circle in the right panel.

The contrast of the PI-PAM comes from the differential signal between two adjacent frames. The differential signal has a nonlinear dependence on the excitation intensity. If the excitation profile can be approximated by a Gaussian function, the full-width-at-half-maximum (FWHM) of the lateral point spread function (PSF) of the imaging system is [15]

$$
F W H M_{r}=\sqrt{\frac{2 \ln 2}{1+b}} w_{e} \approx \frac{0.51}{\sqrt{1+b}} \frac{\lambda_{0}}{N A},
$$

where $r$ is the radial distance from the center of the Airy disk, $w_{e}$ is the Gaussian width of the excitation beam, $b$ is the power dependence of the photobleaching rate on the excitation intensity, $\lambda_{0}$ is the excitation wavelength and $N A$ is the numerical aperture of the objective. The lateral resolution of PI-PAM is sharper than the initial diffraction-limited excitation PSF (Fig. 1b, left panel) by a factor of $\sqrt{1+b}$, where $b$ is the power dependence of the photobleaching rate on the excitation intensity (Supplementary Note 3, Supplementary Fig. 2). Similarly, by sharpening the optical focal zone, PI-PAM provides an axial resolution improvement by a factor of $1 / \sqrt{2^{1 /(1+b)}-1}$ over conventional PAM for point targets.

\section{RESULTS}

\subsection{Sub-diffraction lateral resolution of PI-PAM}

A sharp blade edge coated with hemoglobin was imaged at $532 \mathrm{~nm}$, and the corresponding line spread function was fitted to compute the lateral resolution [15]. With an objective NA of 1.4, conventional PAM achieved a lateral resolution of $200 \mathrm{~nm}$, in agreement with the diffraction limit (Fig. 2a). By contrast, PI-PAM achieved a sub-diffraction lateral resolution of $120 \mathrm{~nm}$, a 1.7-fold improvement. Different materials have different power dependence of bleaching rate and thus different lateral resolution enhancement (Fig. 2b).
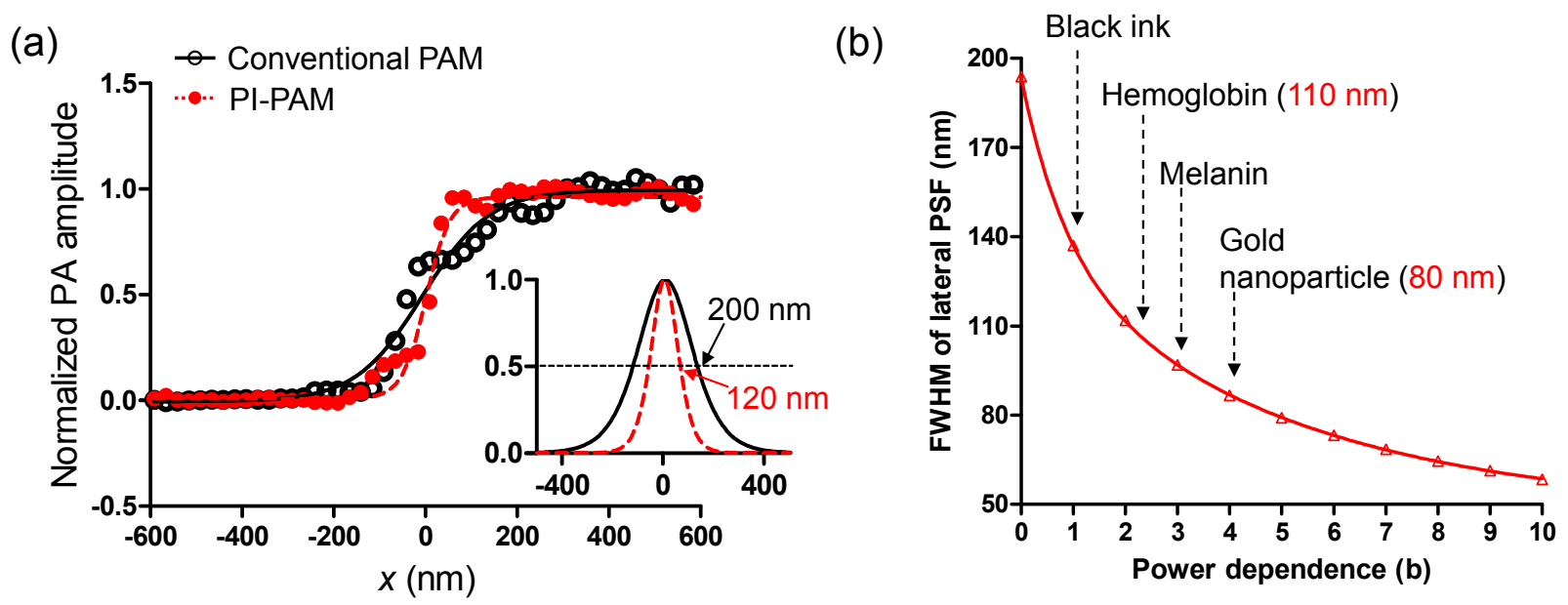

Figure 2. Lateral resolution enhancement by PI-PAM. (a) Edge spread function of conventional PAM and PI-PAM, using a sharp blade edge coated with hemoglobin. The derived line spread functions (inset) show a lateral resolution of $200 \mathrm{~nm}$ for the conventional PAM and $120 \mathrm{~nm}$ for PI-PAM. (b) The lateral resolutions of PI-PAM as a function of the power dependence of photobleaching rate on the excitation intensity. The typical PA absorbers are also marked with their corresponding lateral resolutions.

\subsection{Optical sectioning of PI-PAM}

The nonlinear nature of the PI-PAM signal enables optical sectioning. Compared with the in-focus absorbers, the out-offocus absorbers are bleached less (Fig. 3a). Therefore, the differential signal effectively blocks the out-of-focus contributions and provides optical sectioning for PI-PAM. We quantified the sectioning by using a $\sim 150 \mathrm{~nm}$ layer of 
dried hemoglobin (Fig. 3b). The FWHM of the signal profile suggests a sectioning capability of $370 \mathrm{~nm}$ after deconvolution with the absorption profile of the sample, close to the expected value $(320 \mathrm{~nm})$.

(a)

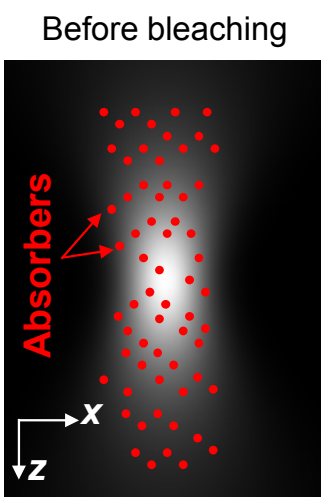

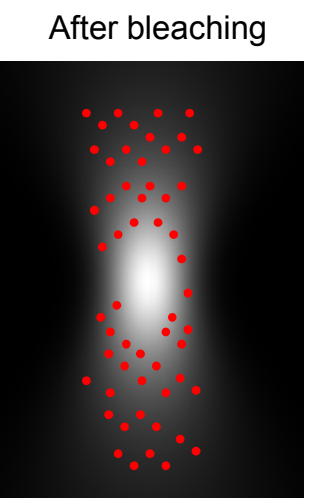

PI-PAM

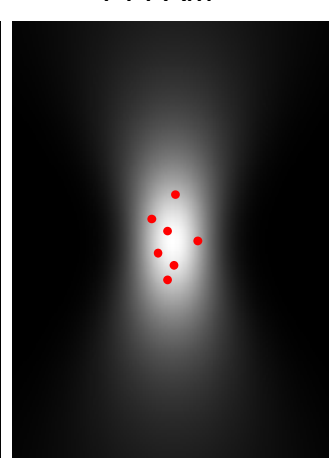

(b)

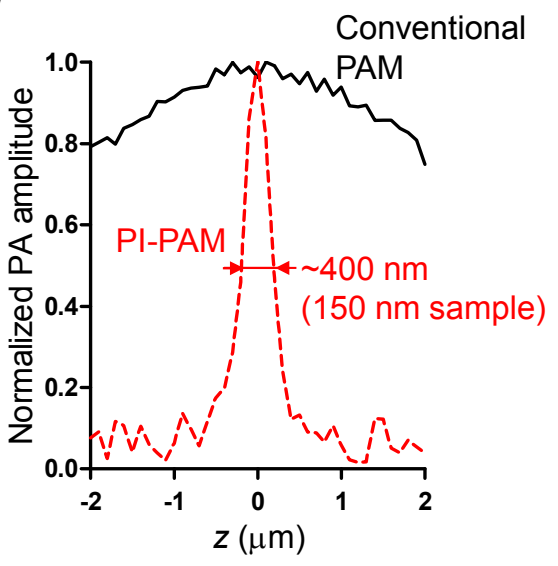

Figure 3. Optical sectioning capability of PI-PAM. (a) Illustration of the optical sectioning capability provided by PI-PAM. Since the photobleaching rate depends on the laser intensity, the absorbers within the optical focal zone $(\sim 400 \mathrm{~nm})$ are bleached more than the absorbers out of the focal zone. The differential signal of the before- (left) and after-bleaching (middle) images largely removes the out-of-focus contributions, thus providing optical sectioning in PI-PAM, which is not possible in conventional PAM for large targets. (b) The axial point spread function measured on a thin-layer hemoglobin sample, showing an optical sectioning capability of $\sim 400 \mathrm{~nm}$ for PI-PAM.

\subsection{PI-PAM of non-fluorescent and fluorescent samples}

We demonstrate the super-resolution imaging capability of PI-PAM on both non-fluorescent and fluorescent samples (Fig. 4). We first compared conventional PAM and PI-PAM images of 150-nm-diameter gold nanoparticles. Two closely located nanoparticles, $270 \mathrm{~nm}$ apart, were clearly resolved by PI-PAM but barely resolved by conventional PAM (Fig. 4a). Deconvolution of the line profile using the Gaussian approximation results in an effective lateral resolution of $80 \mathrm{~nm}$ for PI-PAM. The donut shape of the red blood cells can also be much better resolved with PI-PAM (Fig. 4b).

(a)
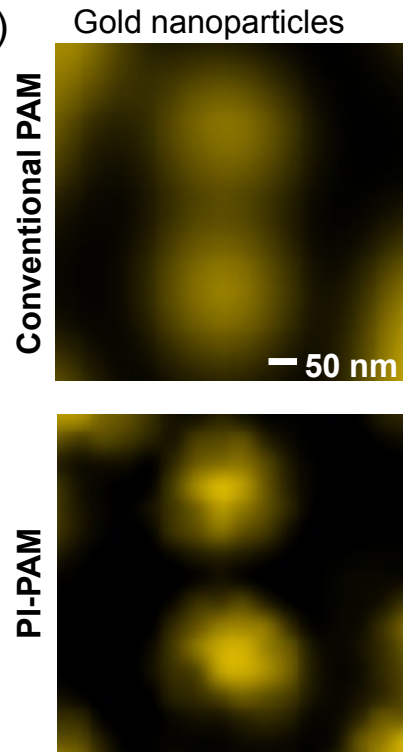
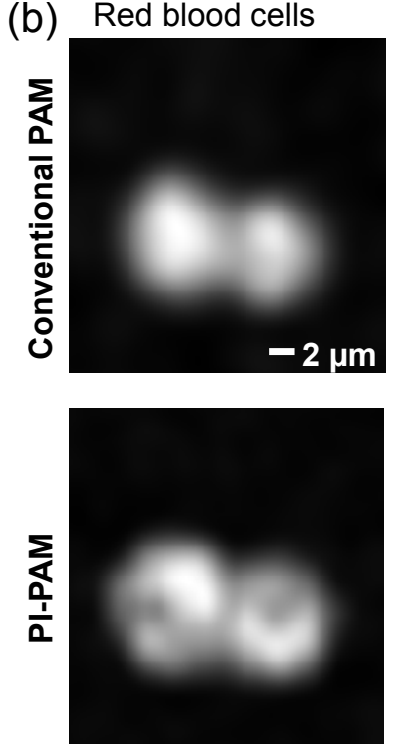
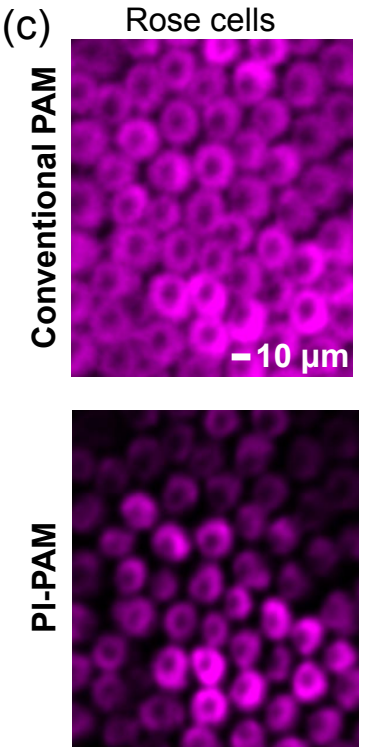

Figure 4. Sub-diffraction PI-PAM of non-fluorescent and fluorescent samples. Conventional PAM (top row) and PI-PAM (bottom row) of (a) 150-nm-diameter gold nanoparticles, $270 \mathrm{~nm}$ apart, (b) red blood cells with donut shape, and (c) rose pedal epidermal cells. The PI-PAM images are clearly superior in resolution over conventional PAM. 
We then applied PI-PAM to sub-diffraction imaging of live rose petal epidermal cells at $570 \mathrm{~nm}$. Pink anthocyanins in cell vacuoles provided the absorption contrast for PA imaging, and they are also commonly used for fluorescence imaging (Fig. 4c). Compared with the conventional PAM images, the PI-PAM images greatly block the out-of-focus signals and sharpen the in-focus signals (Fig. 4c). With $z$-scanning, PI-PAM has achieved three-dimensional subdiffraction resolution.

\section{CONCLUSIONS AND DISCUSSION}

In summary, we have demonstrated three-dimensional sub-diffraction photoacoustic imaging by using the photobleaching effect which is otherwise regarded harmful. The resolution enhancement is attained without complicating the imaging system or relying on specially designed contrast agents.

We note that PI-PAM can be applied to virtually all materials commonly used in biological studies, since photobleaching is a very common phenomenon [16]. If permanent photobleaching is a concern, the dynamics of photoswitchable chromophores can be used instead. Further, the same principle of PI-PAM can be readily transferred to fluorescence microscopy $[11,17,18]$. Since the resolution enhancement gained by the photobleaching effect is complementary to other super-resolution mechanisms and it does not require modification to the existing systems, the principle of PI-PAM can be incorporated into other super-resolution methods to further improve their resolving powers $[8,19,20]$. These merits collectively suggest that PI-PAM holds great potential for broad applications in both fundamental biological study and clinical practice.

\section{ACKNOWLEDGMENTS}

This research was supported by the NIH Grants DP1 EB016986 (NIH Director's Pioneer Award), R01 EB008085, R01 CA134539, U54 CA136398, R01 CA157277, and R01 CA159959. L. V. Wang has a financial interest in Endra, Inc., and Microphotoacoustics, Inc., which, however, did not support this work.

\section{REFERENCES}

[1] Hell, S. W., "Toward fluorescence nanoscopy," Nature Biotechnology. 21(11), 1347-1355 (2003).

[2] Lippincott-Schwartz, J. and Manley, S., "Putting super-resolution fluorescence microscopy to work," Nature Methods. 6(1), 21-23 (2009).

[3] Huang, B., Bates, M., and Zhuang, X. W., "Super-Resolution Fluorescence Microscopy," Annual Review of Biochemistry. 78, 993-1016 (2009).

[4] Flors, C. and Earnshaw, W. C., "Super-resolution fluorescence microscopy as a tool to study the nanoscale organization of chromosomes," Current Opinion in Chemical Biology. 15(6), 838-844 (2011).

[5] Fernandez-Suarez, M. and Ting, A. Y., "Fluorescent probes for super-resolution imaging in living cells," Nature Reviews Molecular Cell Biology. 9(12), 929-943 (2008).

[6] Dempsey, G. T., et al., "Evaluation of fluorophores for optimal performance in localizationbased super-resolution imaging," Nature Methods. 8(12), 1027 (2011).

[7] Rust, M. J., Bates, M., and Zhuang, X. W., "Sub-diffraction-limit imaging by stochastic optical reconstruction microscopy (STORM)," Nature Methods. 3(10), 793-795 (2006).

[8] Willig, K. I., et al., "STED microscopy reveals that synaptotagmin remains clustered after synaptic vesicle exocytosis," Nature. 440(7086), 935-939 (2006).

[9] Jacobson, K., et al., "Fluorescence Photobleaching in Cell Biology," Nature. 295(5847), 283-284 (1982).

[10] Rasnik, I., McKinney, S. A., and Ha, T., "Nonblinking and longlasting single-molecule fluorescence imaging," Nature Methods. 3(11), 891-893 (2006). 
[11] Gao, L., et al., "Photothermal bleaching in time-lapse photoacoustic microscopy," J Biophotonics. 6(6-7), 543-8 (2013).

[12] Chen, T. S., et al., "High-order photobleaching of green fluorescent protein inside live cells in two-photon excitation microscopy," Biochemical and Biophysical Research Communications. 291(5), 1272-1275 (2002).

[13] Patterson, G. H. and Piston, D. W., "Photobleaching in two-photon excitation microscopy," Biophysical Journal. 78(4), 2159-2162 (2000).

[14] Wang, L. H. V. and Hu, S., "Photoacoustic Tomography: In Vivo Imaging from Organelles to Organs," Science. 335(6075), 1458-1462 (2012).

[15] Yao, J. J., et al., "Photoimprint Photoacoustic Microscopy for Three-Dimensional Label-Free Subdiffraction Imaging," Physical Review Letters. 112(1), 014302 (2014).

[16] Launer, H. F., "Photobleaching[mdash]a Common Phenomenon," Nature. 218(5137), 160161 (1968).

[17] Gao, L., et al., "Photobleaching imprinting microscopy: seeing clearer and deeper," Journal of cell science. 126(23), 142943 (2013).

[18] Yao, J. J., et al., "Reversibly switchable fluorescence microscopy with enhanced resolution and image contrast," Journal of Biomedical Optics. 19(8), 086018 (2014).

[19] Gustafsson, M. G. L., "Nonlinear structured-illumination microscopy: Wide-field fluorescence imaging with theoretically unlimited resolution," Proceedings of the National Academy of Sciences of the United States of America. 102(37), 13081-13086 (2005).

[20] Bates, M., et al., "Multicolor super-resolution imaging with photo-switchable fluorescent probes," Science. 317(5845), 1749-1753 (2007). 